\title{
Atypical Case of Pityriasis Rosea in a Child Following Streptococcal Erythema Nodosum
}

\author{
Anca Chiriac1,2,3, Piotr Brzezinski ${ }^{4}$, Cristian Podoleanu5, Simona Stolnicu 6,7 \\ ${ }^{1}$ Nicolina Medical Center, Department of Dermatology, Iași, Romania \\ 2 Apollonia University, Iași, Romania \\ 3 P. Poni Research Institute, Romanian Academy, Iași, Romania \\ 4 Department of Dermatology, 6thMilitary Support Unit, Ustka, Poland \\ 5 Department of Internal Medicine, University of Medicine and Pharmacy, Tîrgu Mures, Romania \\ ${ }^{6}$ Department of Pathology, University of Medicine and Pharmacy, Tîrgu Mureș, Romania \\ 7 Histopat Laboratory, Tîrgu Mureș, Romania
}

\section{CORRESPONDENCE}

\section{Cristian Podoleanu}

Str. Gh. Marinescu nr. 1

540099 Tîrgu Mureș, Romania

Tel: +40744573784

Email: podoleanu@me.com

\section{ARTICLE HISTORY}

Received: November 11, 2017

Accepted: November 25, 2017

Anca Chiriac • Str. Universitătii nr. 16, 700115 lași, Romania. Tel: +40 232267801

Piotr Brzezinski • Department of Dermatology, 6th Military Support Unit, os. Ledowo 1N, 76-270 Ustk Poland. Tel: +48 692121516

Simona Stolnicu • Str. Gheorghe Marinescu nr. 38 540139 Tîrgu Mures, Romania. Tel: +40 265215551

\begin{abstract}
Introduction: Pityriasis rosea (PR) is a widespread skin erythemato-squamous eruption, occurring mostly in young adults. Case presentation: A 9-year-old patient presented with multiple lesions developed after streptococcal pharyngitis and erythema nodosum diagnosed and treated with penicillin prior to the PR. Conclusion: This unique case should be considered a coincidence of two consecutive diseases.
\end{abstract}

Keywords: infection, purpura, therapy, pityriasis rosea, erythema nodosum

\section{INTRODUCTION}

Pityriasis rosea (PR) is a skin disorder characterized by higher prevalence in healthy young adults, occurring mostly during spring and fall, with self-limited course, rare relapses, associated with a possible viral etiology: human herpes virus 6 and human herpes virus 7, Epstein-Barr virus and cytomegalovirus. ${ }^{1,2}$

In classical cases of PR, a "herald" lesion precedes the eruption, being described as a larger erythematous plaque sharply demarcated and covered by fine scales, followed after a couple of days by numerous erythematous scaly plaques distributed mostly on the trunk and proximal extremities. The typical distribution of the lesions along the cleavage lines of the skin creates the "Christmas tree" pattern characteristic for pityriasis rosea. Each plaque, of different size, is well delineated and covered by thin scales with a distinctive feature - a "collarette" scale. The lesions heal spontaneously in 6-8 weeks; in rare cases, they can be accompanied by discrete pruritus. 

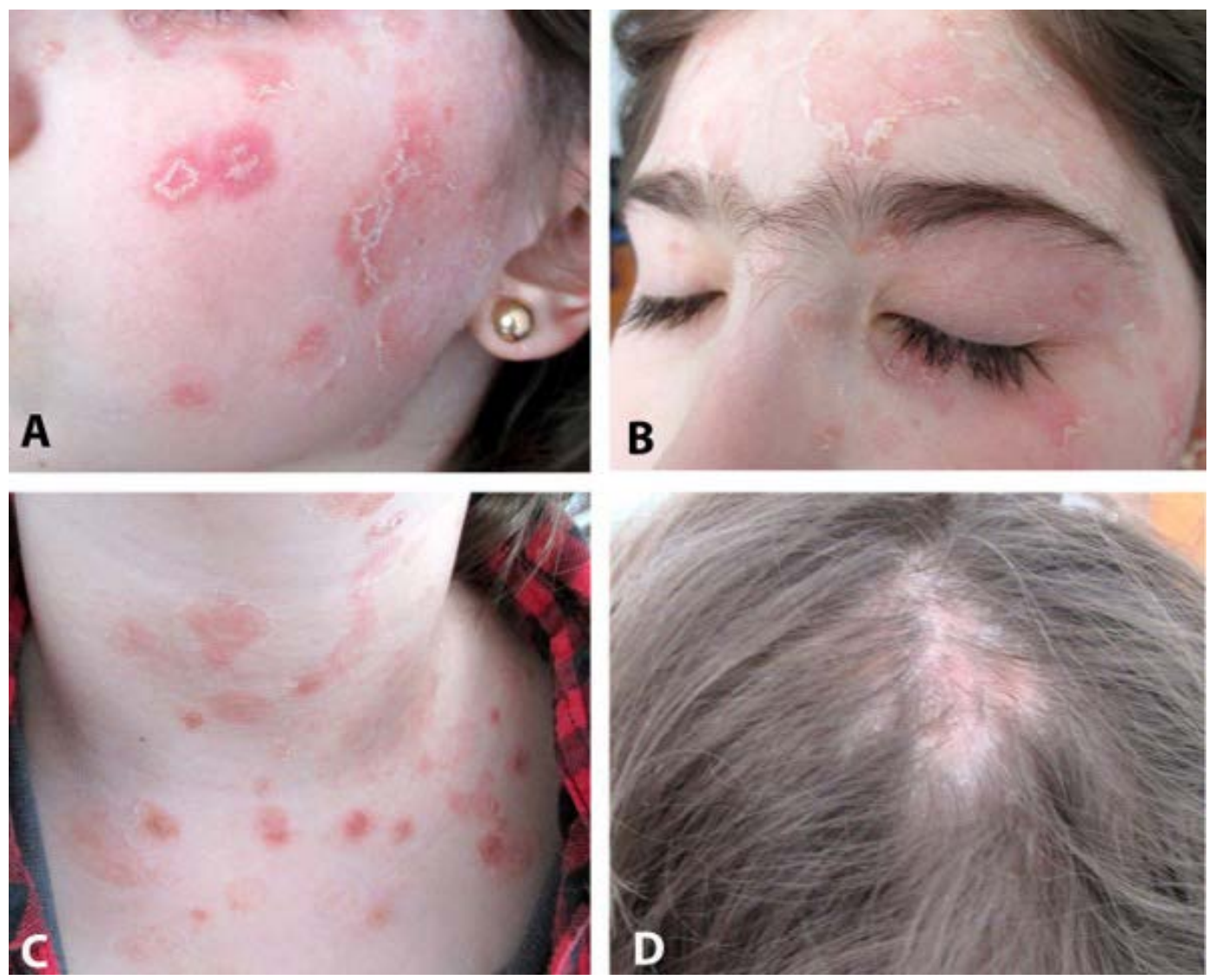

FIGURE 1. Erythematous scaly plaques scattered on the face (A and $\mathbf{B})$, neck and the presternal area (C), and on the scalp (D).

It is not a rare disease so, apart from classical type, many so-called atypical forms have been described, and thus pityriasis rosea can become an enigma or a surprise. ${ }^{1}$

\section{CASE PRESENTATION}

A 9-year-old girl was seen in consultation at the Dermatology Department for multiple erythematous scaly plaques scattered on the face, scalp, neck, and the presternal area. The patient had been hospitalized 6 weeks prior to this consultation for erythema nodosum on the inferior limbs. Family members described the presence of tender, erythematous, subcutaneous nodules on the anterior surface of the lower extremities accompanied by fever, malaise, and upper respiratory tract infection. Nasopharyngeal and throat swabs were analyzed for beta-hemolytic streptococci and the result revealed group A beta-hemolytic Streptococcus. Apart from high anti-streptolysin O (ASO) titer, all usual lab parameters were within normal limits, and chest $\mathrm{X}$-ray was normal.

Streptococcal pharyngitis and erythema nodosum were admitted as diagnosis, and intramuscular benzathine peni- cillin $\mathrm{G}$ was recommended for 10 days, with positive results: clearance of the skin lesions and negative throat culture. The child was closely followed-up by a pediatrician, and the lesions completely healed within the next 4 weeks.

Six weeks after the previous hospitalization, she was referred to the Dermatology Unit for asymptomatic erythematous scaly plaques scattered on the face, scalp, neck, and presternal area (Figure 1, A-D). She was apparently in good condition, with no fever and no systemic complaints, and clinical examination failed to reveal pathological findings. The total blood count, blood chemistry, and erythrocyte sedimentation rate were within normal limits. $\mathrm{KOH}$ examination and fungal culture did not reveal any fungal elements. Serological test for syphilis (rapid plasma regain, RPR) was negative. No drug intake was admitted at the moment of current hospitalization.

A skin biopsy was taken, and the histological report described hyperkeratosis, focal parakeratosis, discontinuous hypergranulosis, moderate irregular acanthosis, exocytosis, spongiosis, and minor lymphocytic infiltrate in the superficial dermis. A diagnosis of pityriasis rosea was established, based on the clinical examination and histo- 
logical report, excluding guttate psoriasis, dermatophytosis, secondary syphilis, lichen planus, or drug eruption. Emollients have been prescribed and the child was seen weekly for the following 6 weeks. No other lesions have been developed after the treatment, and previous lesions faded slowly. Informed consent for the publication of the case was obtained from the child's mother.

\section{DISCUSSION}

PR commonly affects adolescents and young adults, cases reported in children being rarer. ${ }^{1}$ Although $\mathrm{PR}$ is not a rare disease, its etiology is still not completely understood. Infectious agents (human herpes virus 6 and human herpes virus 7, Epstein-Barr virus and cytomegalovirus), drugs (ACE inhibitors, hydrochlorothiazide, captopril, barbiturates, gold, metronidazole, allopurinol, nimesulide, rituximab) and environmental factors have been incriminated. ${ }^{1-4}$

Apart from the classical plaque type, atypical morphological variants of PR exist: urticarial, vesicular, bullous, lichenoid, purpuric, erythema multiforme-like, and exfoliative type. ${ }^{5,6} \mathrm{~A}$ history of preceding respiratory or gastrointestinal infection and isolation of beta-hemolytic streptococcus in throat cultures were confirmed in PR.7

\section{CONCLUSIONS}

The present case of $\mathrm{PR}$ is distinctive for the following elements: occurrence at a young age (9 years), plaque-type PR lesions distributed on the scalp, face, neck, and prester- nal area, no "herald" lesion, and diagnosed 6 weeks after streptococcal pharyngitis and erythema nodosum treated with intramuscular benzathine penicillin G.

Among previously reported cases of PR we have not found any information concerning a possible link between streptococcal erythema nodosum preceding PR or any other similar reported cases. In these circumstances, we should probably consider the coincidence of two consecutive diseases rather than a probable trigger factor for PR. More than 200 years after its first description, PR remains a clinical entity with many unclear facets.

\section{CONFLICT OF INTEREST}

Nothing to declare.

\section{REFERENCES}

1. Rebora A, Drago F, Broccolo F. Pityriasis rosea and herpesviruses: facts and controversies. Clin Dermatol. 2010;28:497-501.

2. Canpolat Kirac B, Adisen E, Bozdayi G, et al. The role of human herpesvirus 6, human herpesvirus 7, Epstein-Barr virus and cytomegalovirus in the aetiology of pityriasis rosea. J Eur Acad Dermatol Venereol. 2009;23:1621.

3. Brar BK, Pall A, Gupta RR. Pityriasis rosea unilateralis. Indian J Dermatol Venereol Leprol. 2003;69:42-43.

4. Atzori L, Pinna AL, Ferreli C, Aste N. Pityriasis rosea-like adverse reaction: review of the literature and experience of an Italian drug-surveillance center. Dermatol Online J. 2006;12:1.

5. Chhabra N, Singal A, Pandhi D. Pityriasis rosea unilateralis with atypical morphology. Int J Dermatol. 2014;53:e92-e93.

6. Bhalla N, Tambe S, Zawar $\mathrm{V}$, et al. Localized purpuric lesions in a case of classical pityriasis rosea. Indian J Dermato/ Venereol Leprol. 2014;80:551553.

7. Ozyürek GD, Alan S, Cenesizoğlu E. Evaluation of clinico-epidemiological and histopathological features of pityriasis rosea. Postepy Dermatol Alergol. 2014;31:216-221. 